\title{
World Wide Web Adapted Geometric Model in the Context of Functional Design
}

\author{
F. Danesi, C. Dartigues, Y. Gardan \\ CMCAO, IFTS, Charleville-Mézières, France \\ Em:\{danesi,dartigues\}@netcourrier.com,gardan@infonie.fr \\ E. Perrin \\ CMCAO, Metz university, Metz, France \\ Em: perrin@lrim.univ-metz.fr
}

Keywords World Wide Web, Functional Design, Geometric Modelling

\begin{abstract}
Our goal is to create a CAD system dedicated to WWW. Offering a CAD system over the WWW is a viable topic that is still too difficult to handle. The main problem is to transfer CAD system data, which are classical huge. Unfortunately this transfer is time and web-line consuming and no specific method exists. This paper begins with a survey of the current field of algorithm reducing data volume, in order to transfer less data on the web. Since they are not dedicated to CAD model, which are still huge after treatments, we propose three transmission methods specific to each main model used in CAD: CSG, octree and B-Rep. We show their limits, which can be pushed back by a functional model.
\end{abstract}

\section{INTRODUCTION}

Current CAD-CAM systems are efficient in their favourite field: geometric modelling. They propose designed interfaces to build parametric objects and to act on shapes. If present systems help the designer when the shape of the object is known, it is regrettable that only parametric and variational designs take into account the designer's know-how. In practice, design intent and methodology are totally given up at the beginning of the computer use. This arises numerous basic drawbacks. The user has to start with geometric constraints and shape's creation and nothing shows that the geometric model is adequate to functions and constraints of the 
specifications. No interface software tool is available to capture user's know-how and functions of the specifications.

In that context, a tendency rises in order to define less monolithic systems that work with interoperability: virtual companies have emerged due to the World Wide Web extend and communications between server and host (or client) are numerous. For a time, only bitmaps of a 3D scene have been transmitted to the client through compression algorithms. In that case, the server made computer calculations and the client was only showing the results. Nowadays, technique changes: it may be interesting that the client could make specific operations by its own and relieve the server. This means that a geometric description of the 3D scene is then necessary to the client. Unfortunately, geometric description is huge. Thus the entire description can not be transmitted to the client because of communications time. If the image compression has been largely studied, geometric compression research (including algorithmic geometry and data compression) is recent and available papers deal with triangle mesh compression.

\section{FUNCTIONAL WWW CAD SYSTEM}

The will to capitalise the know-how and to reduce times of production make that one now truly approaches the CAD-CAM systems in the optics of a functional modelling. Our goals are to assist the designers during the earlier stages of the product design and to create a system dedicated to any kind of firm. The latter needs to take into account the level of computer knowledge of all the employees, who are potential users of the system. The interface will have to integrate intuitive notions in order to capture the user's know how. However the current systems are still based on geometry and in order to achieve the wished goals, it is necessary to delay computations and to introduce higher semantic level concepts [1].

Some commercial firms try to use WWW to distribute their system but commercial CAD systems are heavy to manage, tedious to understand and to learn and require powerful computers. We want to develop a new approach to implement more ergonomic CAD systems, using WWW as a tool. The firm will not have any longer the responsibility of the system management. This will be done by the company that distributes the system on internet; the user will just have to load software components he needs to work; computations will be done by the server computer so time responses will be related only to network and not to host processor's power. Thus, the small companies will be able to reach technologies that were hitherto inaccessible for lack of financial, material and intellectual means. 
All of this implies to create a functional model and an adequate interface, to manipulate the model, to translate it into geometrical data to obtain a shape and to transfer it from the server to the host computers. As it is difficult to detail functional and WWW problems at the same time, we will highlight problems resulting from the use on Internet with a geometrical model in the next section. For further details on functional modelling and functions to shape translations, see $[2,3]$.

\section{TRANSFER OF GEOMETRICAL DATA FOR WWW}

Our goal is to create a CAD system available on internet with the same characteristics as a classical one, with powerful tools (for example to draw up an estimate of mechanical parts) and with more specific ones useful to a given profession (such as the one to calculate the shape of a mould in foundry [4]). Obviously, the system responses to the user's interactions must be similar to a classical CAD system ones. This problem relies on current network flow, computer memory space and huge size of used geometrical data. This study consists in managing the transfer of geometrical information between the host and the server as quick and transparent as possible. In the next sections, we will show that the methods encountered in the bibliography can not solve problems coming from these constraints and we will suggest solutions for the main CAD models: historic construction, octree and boundary representation.

\section{Overview}

In order to work, the CAD system user on a distant host has to receive some topological and geometrical data contained in the model. The product model may become rapidly consequent and it can not be sent as it is.

The compression methods $[5,6,7,8,9,10,11,12]$ permit to reduce the data volume. They are usually used in virtual reality and in medical imaging but they can be adapted for CAD because they commonly work with a mesh. In that case, the CAD model must be translated into a mesh before the method runs [9], which is often realised in a CAD system. Compression methods proceed in two steps. First, they code the topology of the mesh, which gives a list of vertices sorted by proximity. Secondly, for each vertex of the list, they predict its position according to the previous vertices. The vertex code is obtained by subtracting the real position with the predicted one. It can be noted that good results have been obtained in finite element meshes to reduce time calculus by reducing the mesh. In our case, compression 
methods are not adequate because CAD model must be translated into a mesh, which spends time. Moreover data volume remains large after compression and must be sent once: the user has to wait end of transmission to work and he receives the entire model even if he does not need it.

The simplification methods $[13,14,15,16,17,18]$ send the model in several times and then give an approximate view of the model from the first transmission. The model is then refined step by step. As compression methods, they work on meshes. Before transmission, the mesh is simplified recursively until it reaches the desired level of approximation that is fixed at the beginning of the process. Each iteration gives a level of simplification and affects the model homogeneously. In that case, the user's waiting is compensated by a progressive view of the model but he still can not work before the end of the reconstruction.

The selective simplification methods $[19,20,21,22]$ are more convenient because they adapt the model reconstruction to the user's needs. Parts of the model are chosen according to the user point of view and are firstly reconstructed. Unfortunately modifications he may realise can alter parts of the model that have not been yet transmitted. Model maintenance problems may occur between the host and the server computers. It is also damageable that selective simplification methods rely on meshes.

Nevertheless, selective simplification methods give a way of thinking to an intelligent system taken into account the user's will, that is here reduced to point of view. However, this intelligence may increase if more information on the user's know-how are given to the interface. The latter is then able to interpret and control the will instead of only capturing it: if a modification alters a part of the model that has not been yet transmitted, then the interface can detect it and anticipate the transmission. In that case, the interface has no longer this passive role of translator it used to have between user actions and system orders. This purpose would not be done without a functional model. As we have mentioned it, we only focus at the moment on geometrical models. So we restrict our next study to the three mains geometrical model used in CAD in order to choose the most convenient.

\section{Dedicated methods to geometrical CAD models}

The methods of the section 0 work with meshes. However, the three main geometrical models used in CAD are constructive solid geometry, octree and boundary representation. They could be translated into meshes but it is time consuming and more important, information losing. Because of the lack of study about transfer with the three main models, we then propose three dedicated methods to them and show their limits. 


\section{Our method to transfer Constructive Solid Geometry}

CSG (Constructive Solid Geometry) is a graph, whose nodes are Boolean operations and leaves are simple geometric primitives. CSG represents the model implicitly. Historic construction is the same way of representation as CSG but it is used with form features (like slots, bosses or blind holes): instead of primitives, features are used. The order of features defines the order of design. Its advantage is then to contain higher level information that corresponds to the user's design intent. That is the reason why we prefer studying historic construction rather than CSG.

To add a feature, the user has to view the object he is constructing in order to position the feature. This means that the historic construction has to be translated into an explicit model, in general into a boundary representation. The position of the feature is, for example, relative to a face of the object that is selected by the user. Then the form feature is instantiated by a generic procedure, whose parameters are defined by the user and the new object is created. Only the kind of form features (which can be coded by a number), its relative position and their associate parameters are necessary to transfer. The relative position is related to the explicit model. As the transfer must be as quick as possible, we want to avoid sending the explicit model. To realise this, the relative position has to be coded as an absolute position, which is totally defined by the viewing parameters (eye's coordinates and sighting axis co-ordinates) and the mouse co-ordinates.

The historic construction is compact and is obviously rapid to transfer. Nevertheless, the explicit model has to be reconstructed just after the transmission, which costs time. As seen in the overview (in section 0), simplification and above all selective simplification can be used to reduce this problem. Selective simplification is totally adequate to our case because it takes into account the user point of view, which has been saved for the transmission of the model. The idea is then to select only the form features with a close point of view of the host current one. This operation raises to a major problem: selecting the form features according to the point of view neglects interference between features. In that case, adding a new feature may perturb the design intent and then arises to a wrong solution.

\section{Our method to transfer octree}

Octree is not an exact model but it is often used when it facilitates graphic treatments. It is a space recursive decomposition into black, white or grey cubes. The colour of the cube expresses its belonging (black) or not (white) to the object. A grey cube has to be subdivided. So, its main drawback is that it is memory space consuming. To reduce this problem, we 
propose a method to calculate the biggest black boxes (instead of cubes) at each decomposition step. The principle of this method is to subdivide a box according to [Ox) axis, then to subdivide independently the two resulting boxes according to [Oy) axis and to iterate with the four resulting boxes according to $[\mathrm{Oz})$ axis. Subdivision according to an axis is realised by choosing the best section plan in order to obtain the biggest black box. Subdivision can be avoided when it may start with a black or white box.

The created structure is then a degenerated octree. It is more compact than a classical one and then is quicker to send even if it is more time consuming to build. The degenerated octree is naturally adapted for selective simplification. At the beginning of the session, a tree corresponding to a low level of decomposition is sent to the host. In visualising it, the host is able to detect which boxes are visible and then asks the server their next level of decomposition in order to obtain a more precise visualisation.

However, even if the user views exactly his object, he could not modify it before he receives the exact model too, which could not be reconstructed from the octree without losing information. So it has to be transferred.

\section{Our method to transfer Boundary representation}

B-Rep (Boundary representation) is a structure, which contains all the vertices, edges and faces of the object that it represents exactly (when it is polyhedral) and explicitly. We have shown, in the two previous sections, that an exact representation is required in a CAD system. Unfortunately, BRep is often a huge structure and it is inconceivable to send it once. We have overviewed that the methods in section 0 are not adapted to our problem. To reduce the user delay time and to let it work as soon as possible, only the visible faces can be sent to the host. The number of visible faces is relative to the complexity of the object according the user point of view. In general, the number of visible faces is very less than the number of total faces but, for the reason mentioned above, this can be consequent anyway.

To avoid this problem, we propose to send first an image to the host instead of the model. This principle can be used each time the user changes of point of view. This can be done in three steps as following: first, the server asks the point of view to the host. Secondly, the server calculates the corresponding image: this can be done actually fast in using appropriate processors. Finally, the server sends the image and the host shows it.

The needed part of the model can be sent later during the time that the user is watching to the object. If this delay is too short, the user may interact before the transmission ends. In that case, the needed faces, that are dependent of the interaction, may have been received or not. If not, the appropriate faces must be selected by the server and sent to the host. A local 
image can be then calculated and the global image can change following the user interaction. But finding the adequate faces (and then finding the parts of the image that must be recalculated) is a main problem because the faces are not only the ones directly concerned by the interaction (the user shows a face) but also the ones modified by the interaction (some faces may become visible because of the modification of another). In a B-Rep, only topological and geometrical information is saved and this problem concerns higher level information and calls for a functional model: for example, it would be easier to know that a given face is behind another face [23].

\section{CONCLUSION}

Our goal is to create a CAD system dedicated to WWW with the same properties as a classical one. Such an aspiration needs to solve data transfer time, quantity of transferred data, etc. The current methods in the state of the art dedicated to rapid data transfer concern compression and medical imaging. Unlucky, no method is specific to CAD system because this problem raises with particular models such as CSG, octree and boundary representation that are not used for compression and medical images. We have shown that CSG and octree, even if they can be compact, are inapt to let the user works because they are not exact representation. Boundary representation, that is an exact representation is unfortunately huge and can not be send in totality. If images are used to reduce data quantity in that case, this treatment needs higher level information, that boundary representation does not possess. Such information would be saved in a functional model. However, even if only geometric models are used, we have shown that by taking into account the inherent properties of each one, it is possible to obtain good results using, basically, the three following directions. First, modify the model in order to adapt it to the future treatments. Secondly, take the user point of view as a fundamental information to know what to send in priority. Finally, send information step by step, enriching the details concerned by the user interaction.

Using a functional model may permit to compress naturally data contained in a geometrical model and perhaps to realise actions corresponding to the user interactions. In that case, we could neglect totally geometrical model in order to use only the functional one. Unfortunately, the latter is still too conceptual to be used in a current CAD system. Thus we are still working with geometrical model in a first time, and our future work will consist in implementing it in our WWW-CAD conceptual system. 


\section{REFERENCES}

[1] Johnson A. L.,Thornton A. C., Fong C. F. (1993). Modelling functionality in CAD: implications for product representations, 9th international conference on engineering design, Hubka eds., The Hague - Netherlands, August 17-19, 1610-1617

[2] Y. Gardan, C. Minich, D. Pallez, E. Perrin, (1999) From Functions to Shapes, Proceedings of EDA'99, Vancouver

[3] Y. Gardan, C. Minich, D. Pallez, E. Perrin (2000) Towards a specifications-to-shape translation tool , Proceedings of TMCE 2000, Delft, April 18-21, 373-382

[4] Gardan Y., Lanuel Y., Pallez D., Vexo F. (2000). Methodology for functions to shape translation tool in foundry, Computers in industry, to be published

[5] Allier P., Laurent N. (1999). Compression et Représentation Echelonnable de Maillages Triangulaires. Proceedings of AFIG, Reims, November 24-26, 89-102.

[6] Chow M. M. (1997). Optimized Geometry Compression for Real-time Rendering, Proceedings of IEEE Visualisation

[7] Hayley M. B., Blake E. H. (1996). Incremental Volume Rendering Unsing Hierarchical Compression, Proceedings of EUROGRAPHICS, Poitiers, 45-55.

[8] Li, J., Kuo C.C. J. (1997) Progressive Compression of 3D Graphic Models, Proceedings of The International Conference on Multimedia Computing and Systems, 135-142.

[9] Masuda H., Ohbuchi R. (2000) Coding topological structure of 3D CAD models, Computer Aided Design, 32(5-6), 367-375.

[10] Taubin G., Rossignac J. (1998) Geometric compression through topological surgery, ACM transactions on Graphics, 17(2), 84-115.

[11] Touma C., Gotsman C. (1998) Triangle mesh compression, Graphics Interface, 26-34.

[12] Rossignac J. (1999) Edgebreaker: Connectivity Compression for Triangle Meshes, IEEE Transactions on Visualisation and Computer Graphics, 5(1), 47-61.

[13] Algorri M.E., Schmitt F. (1996) Mesh Simplification, Eurographics'96, 77-86.

[14] Belblidia S. (1998) Modélisation et visualisation par niveaux de détail de scènes architecturales complexes, $P h D$ Thesis, Institut Polytechnique de Lorraine INPL.

[15] Belblidia S., Perrin J.P. (2000) Simplification de surfaces polygonales complexes MICAD2000, 161-167.

[16] Cignoni P., Montani C., Scopigno R. (1998) A comparison of mesh simplification algorithms, Computer \& Graphics, 22 (1), 37-54.

[17] Fischer A. (2000) Multi-level models for reverse engineering and rapid prototyping in remote CAD systems, Computer Aided Design, 32(1), 27-38.

[18] Rossignac J., Borrel P. (1993) Multi-resolution 3D approximations for rendering complex scenes, Conference on Geometric modelling in Computer Graphics 453-465.

[19] Ciampalini A., Cignoni P., Montani C., Scorpino R. (1997) Multiresolution Decimation based on Global Error, The Visual Computer, 13 (5), 228-246.

[20] Cignoni P., Puppo E., Scorpino R. (1997) Representation and visualisation of Terrain surfaces at variable resolution, The Visual Computer, 13(5), 199-217.

[21] Hoppe H. (1998) Efficient implementation of progressive meshes, Computer \& Graphics, 22 (1), 27-36.

[22] Reinhard K. (1998) Multiresolution representations for surfaces meshes based on the vertex decimation method, Computer \& Graphics, 22 (1), 13-26.

[23] Eustache J., Lanuel Y., Vivian. R (1999) Utilisation de la fonctionnalité d'un objet pour optimiser le calcul d'une image de synthèse, 12èmes journées de l'AFIG, Reims, France. 